\title{
Low Leakage-Current InAsSb Nanowire Photodetectors on Silicon
}

\author{
Michael D. Thompson, ${ }^{*}{ }^{\dagger}$ Aiyeshah Alhodaib, ${ }^{\dagger}$ Adam P. Craig, ${ }^{\dagger}$ Alex Robson, ${ }^{\dagger}$ Atif Aziz, ${ }^{\dagger}$ Anthony Krier,
} Johannes Svensson, Lars-Erik Wernersson, ${ }^{\ddagger}$ Ana M. Sanchez, ${ }^{\text {II }}$ and Andrew R. J. Marshall* ${ }^{\dagger}$

\author{
${ }^{\dagger}$ Department of Physics, Lancaster University, Lancaster, LA1 4YB, United Kingdom \\ ${ }^{\ddagger}$ Electrical and Information Technology, Lund University, Box 118, SE-221 00 Lund, Sweden

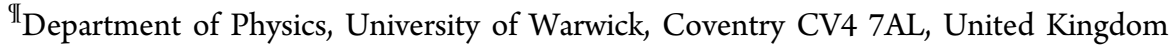

\section{Supporting Information}

\begin{abstract}
Axially doped $p-i-n$ InAs ${ }_{0.93} \mathrm{Sb}_{0.07}$ nanowire arrays have been grown on $\mathrm{Si}$ substrates and fabricated into photodetectors for shortwave infrared detection. The devices exhibit a leakage current density around $2 \mathrm{~mA} / \mathrm{cm}^{2}$ and a $20 \%$ cutoff of 2.3 $\mu \mathrm{m}$ at $300 \mathrm{~K}$. This record low leakage current density for InAsSb based devices demonstrates the suitability of nanowires for the integration of III-V semiconductors with silicon technology.
\end{abstract}
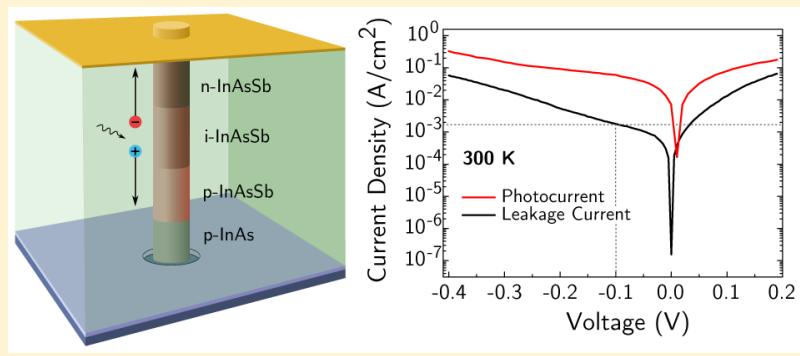

KEYWORDS: Nanowires, midwavelength infrared, photodetector, InAsSb

$\mathrm{T}$ he integration of III-V semiconductors with silicon substrates targets the compelling goal of photonic devices combined with mature silicon technology. ${ }^{1,2}$ However, the heteroepitaxy of bulk III-V materials on silicon substrates results in high defect levels which reduce the crystalline quality and performance of such devices. ${ }^{3}$ Nanowires have been shown to overcome this problem due to rapid strain reduction during growth leading to lower dislocation densities. ${ }^{3,4}$ In this work a shortwave infrared InAsSb nanowire photodetector is grown on silicon, incorporating an axial $p-i-n$ junction and exhibiting an exceptionally low leakage current density of less than $2 \mathrm{~mA}$ / $\mathrm{cm}^{2}$ at $300 \mathrm{~K}$. Significantly, this new technology has a much lower leakage than today's best $\operatorname{InAs}(\mathrm{Sb})$ photodiodes, which have reported leakage current densities around $100 \mathrm{~mA} / \mathrm{cm}^{2}$ at $300 \mathrm{~K}$, despite their increased bandgap and homoepitaxial substrates. ${ }^{5-8}$ In doing so it paves the way for lower noise III$\mathrm{V}$ detectors, which can further benefit from intimate integration with silicon electronics technology.

Nanowires made from InAs and InAsSb are typically grown using metal-organic vapor phase epitaxy (MOVPE) and molecular beam epitaxy (MBE) using the vapor-liquid-solid $(\mathrm{VLS})^{9}$ growth mechanism which uses a metal droplet (either gold or indium) as the nanowire seed. In our work we use a catalyst free method ${ }^{10}$ which begins with lithographic patterning using electron beam lithography $(\mathrm{EBL})^{1}$ followed by wet etching holes in the native oxide of the Si substrate. We then grow the nanowires by MBE and rely on the holes in the oxide to localize the nanowire growth to defined locations. The advantages to this method are the avoidance of gold, which forms trap states in silicon, ${ }^{2,11}$ and simpler growth as there is no need to optimize droplet deposition. The native oxide also prevents material deposition between the nanowires which is typical of growth on unpatterned Si substrates. ${ }^{12}$ Although EBL is slow and costly, nanoimprint lithography ${ }^{13,14}$ will allow for low cost patterning of large area devices. By controlling the site locations, nanowire groups can then be used as individual photonic devices such as pixels in focal plane arrays or ultrasmall detectors.

Figure 1a shows an atomic force microscope (AFM) image of the patterned holes in the resist before wet chemical etching. The holes are around $50 \mathrm{~nm}$ in diameter patterned into a 300 $\mathrm{nm}$ pitch square array. Figure $\mathrm{lb}$ and $\mathrm{c}$ shows scanning electron microscope (SEM) images of InAsSb nanowires grown with this pattern that are on average about $1 \mu \mathrm{m}$ in length and 80 $\mathrm{nm}$ in diameter and the area fraction occupied by the nanowires is approximately $10 \%$. From SEM analysis of various samples we have found that, in cases where a nanowire fails to grow, nearby wires have a greater length variation (circular outline in Figure $1 \mathrm{~b}$ ) and are typically longer because of proximity effects. $^{15}$ This is due to the small pitch of the patterned array being less than twice the diffusion length of indium adatoms on $\mathrm{SiO}_{2}$, which is estimated to be $\sim 750 \mathrm{~nm} .^{16}$ As a result, each nanowire is within the capture area of nearby wires. When a hole is not fully etched and no nanowire grows at that location, the indium adatoms diffuse to neighboring nanowires leading to an increase in their growth rate. In regions of more successful growth the wire length is more uniform as seen in the square outline in Figure 1b. For uniform length distribution in dense arrays, it is therefore crucial that all holes are cleanly etched.

For device applications, it is important that the nanowire is grown directly onto the substrate to provide electrical contact

Received: August 27, 2015

Revised: December 3, 2015

Published: December 16, 2015 


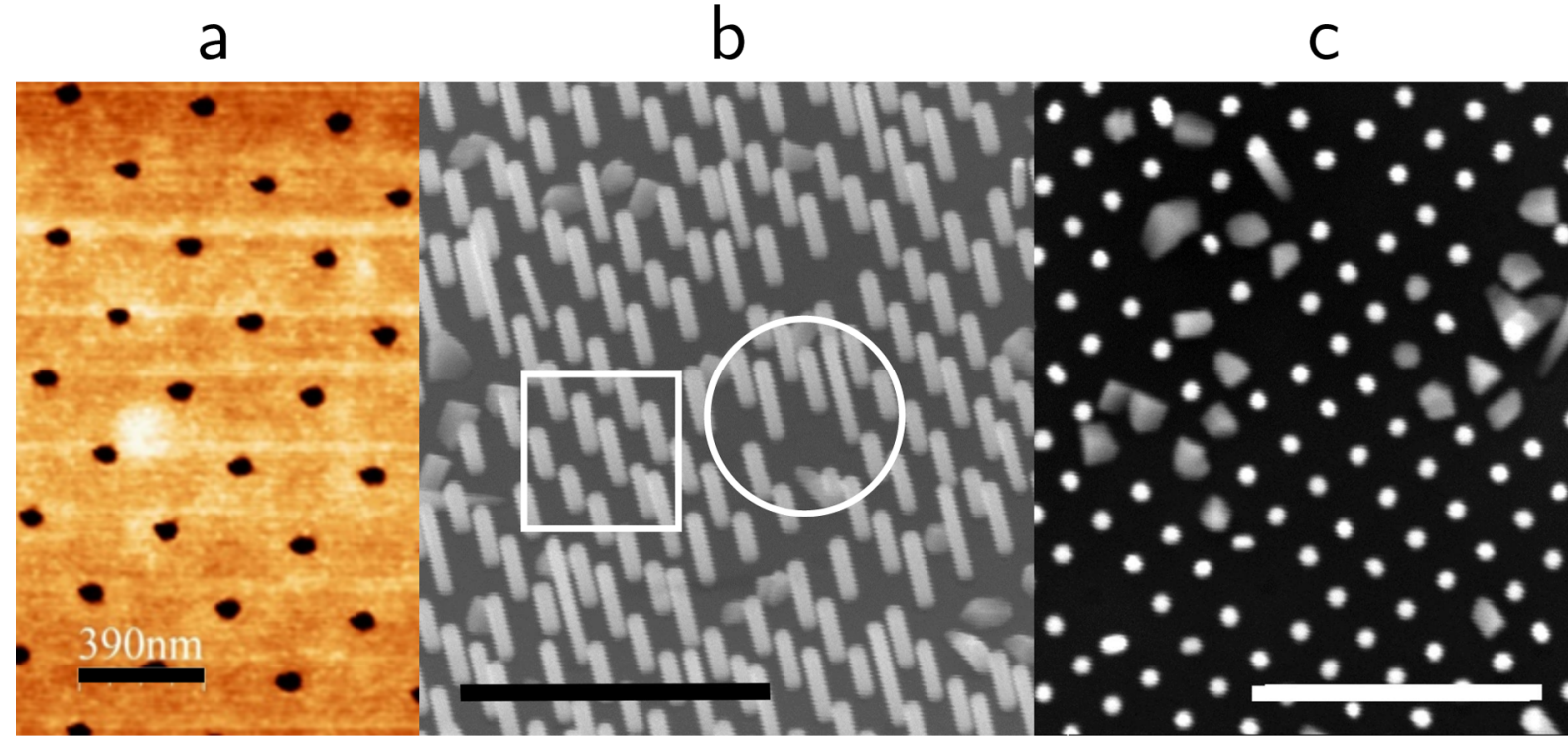

Figure 1. Patterned InAsSb nanowires. (a) Atomic force microscope (AFM) image of the etched holes in the oxide layer on the substrate. Scale bar: $390 \mathrm{~nm}$. (b) SEM image with $30^{\circ}$ tilt of InAsSb nanowires grown in the holes in the oxide layer. The square and circular outlines indicate regions of uniform and nonuniform growth. Scale bar: $2 \mu \mathrm{m}$. (c) Top view SEM image of patterned InAsSb nanowires with highly uniform nanowire diameter. Scale bar: $2 \mu \mathrm{m}$.
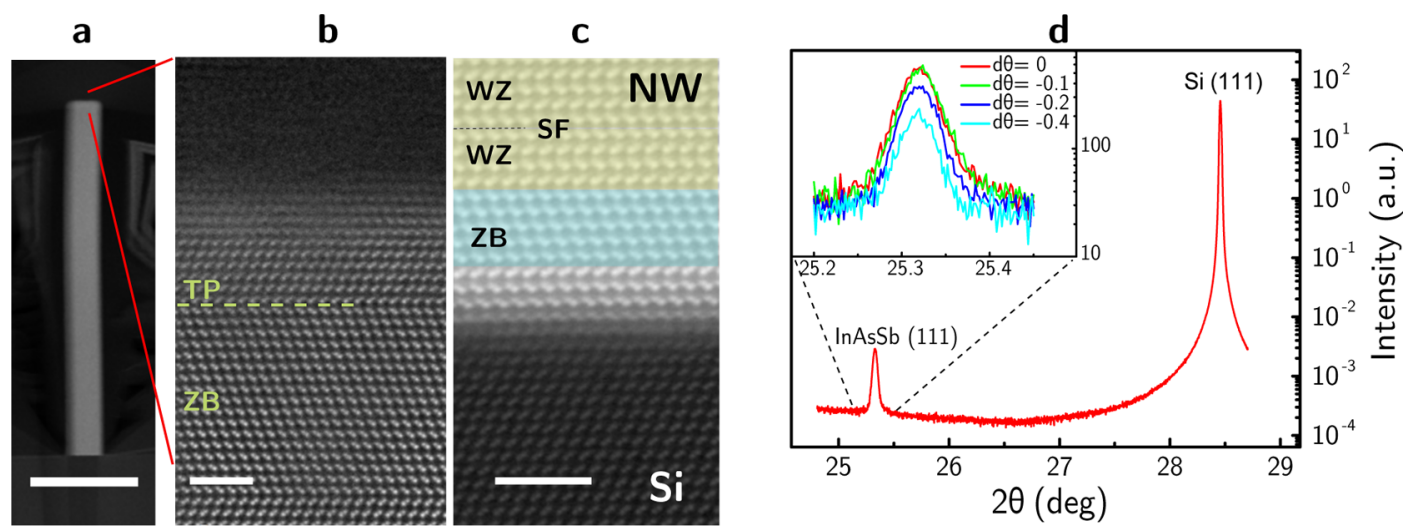

Figure 2. Structural characterization of InAsSb nanowires. (a) Low magnification ADF-STEM image of InAsSb nanowire. Scale bar: $500 \mathrm{~nm}$. (b) The top of the nanowire is flat and shows the multitwinned ZB structure of the InAsSb. Scale bar: $2 \mathrm{~nm}$. (c) High magnification ADF-STEM image corresponding to the InAs nanowire/Si interface shows that the nanowires have grown directly onto the substrate and InAs section shows a mix of WZ (with stacking fault (SF) indicated by the dashed line) and ZB regions. Scale bar: $2 \mathrm{~nm}$. (d) XRD rocking curve of InAs ${ }_{0.93} S_{0.07}$ nanowires on (111) Si substrate. Inset: The InAsSb peak for different $\theta$ offsets which shows only a slight decrease in intensity indicating that the peak originates from the nanowires and not from planar material.

and avoid any series resistance due to the presence of an oxide layer between the nanowire and the substrate. For this work we have grown nanowires using InAs and InAsSb. Figure 2a shows a low magnification annular dark field scanning transmission microscopy (ADF-STEM) image of a nanowire grown on the silicon substrate. Site specific specimen preparation to analyze the nanowire/substrate interface by electron microscopy was achieved using focused ion beam (FIB) lift-out. Figure 2c shows a high magnification ADF-STEM image of the interface between an InAs nanowire and the $\mathrm{Si}$ substrate which shows that the nanowire has grown directly on the substrate. Furthermore, the InAs has the characteristic mix of wurtzite (WZ) and zinc-blende (ZB) phases, ${ }^{17,18}$ while the InAsSb nanowire (Figure $2 \mathrm{~b}$ ) is $\mathrm{ZB}$, as expected. ${ }^{19}$ A polytype phase as seen in InAs can lead to unwanted uncontrollable heterostructures due to the different bandgaps of the $\mathrm{ZB}$ and $\mathrm{WZ}$ phases. ${ }^{20}$ The addition of $\mathrm{Sb}$ forces a $\mathrm{ZB}$ phase with ortho twins which do not change the polarity of the crystal along the nanowire. Compositional analysis (see Supplementary Figure 1) along the length of an InAsSb nanowire revealed that the base of the wire contains a $20 \mathrm{~nm}$ section of polytype InAs suggesting that the incorporation of $\mathrm{Sb}$ is suppressed during the early formation of the nanowire. The reason for this is not yet understood.

A lab-setup XRD system was used to determine the composition of the $\operatorname{InAs}_{1-x} S b_{x}$ nanowires by performing a $2 \theta-\omega$ scan of the 111 reflection of the as-grown sample (see Figure 2d). The peak position corresponds to an $\mathrm{Sb}$ composition of $x=0.07$ which is calculated using the position of the InAsSb peak relative to the substrate peak and is in good agreement with the results from photoluminescence. To ensure that the XRD peak originates from nanowires and not surface growth, several scans with different $\theta$ offsets have been performed showing only a slight decrease in intensity (see 
inset of Figure $2 \mathrm{~d}$ ). Due to the small diameter of the nanowires, their diffraction peaks have a larger in-plane broadening compared to surface growth for which only a small $\theta$ offset is sufficient to decrease the intensity considerably.

Nanowire photodetectors were fabricated by growing an axial $p-i-n$ structure consisting of $250 \mathrm{~nm} p$-type (Be doped) InAs followed by $500 \mathrm{~nm}$-type InAsSb, $500 \mathrm{~nm}$ undoped InAsSb, and finally $500 \mathrm{~nm} n$-type (Te doped) InAsSb. The schematic of a finished device is shown in Figure 3a which highlights the

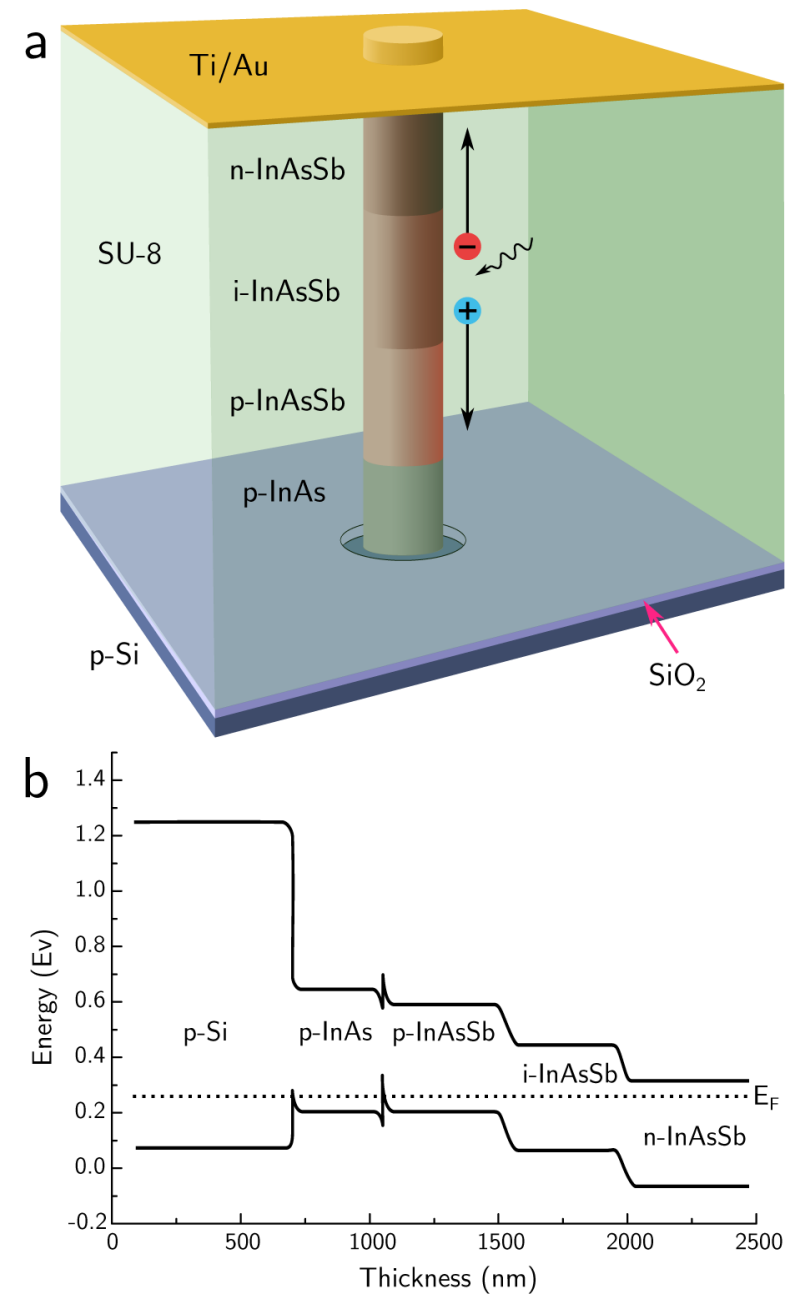

Figure 3. Schematic of $p-i-n$ InAsSb nanowire photodiode. (a) The nanowire consists of a $200 \mathrm{~nm}$ Be doped InAs stub followed by 500 $\mathrm{nm}$ Be doped InAsSb, $500 \mathrm{~nm}$ undoped InAsSb, and $500 \mathrm{~nm}$ Te doped InAsSb. The nanowire is encapsulated by cross-linked SU-8 with $\mathrm{Ti} / \mathrm{Au}$ forming the top contact. (b) Band diagram of the $p-i-n$ photodiode at zero bias.

different sections of the nanowire. Below this, in Figure $3 b$, is a band diagram at zero bias for the full structure. In devices where the junction occurs at the interface between the nanowire and the substrate, the band offsets between the nanowire and silicon can significantly hinder the movement of the carriers. By growing a $p-i-n$ structure, the voltage drop principally occurs across the intrinsic region of the nanowire, from where the photogenerated carriers can drift easily into the majority carrier regions.

The leakage current measured at room temperature is shown in Figure 4. At a typical detector operational bias of $-0.1 \mathrm{~V}$, the reverse leakage current was $18 \mathrm{pA}$, which corresponds to a

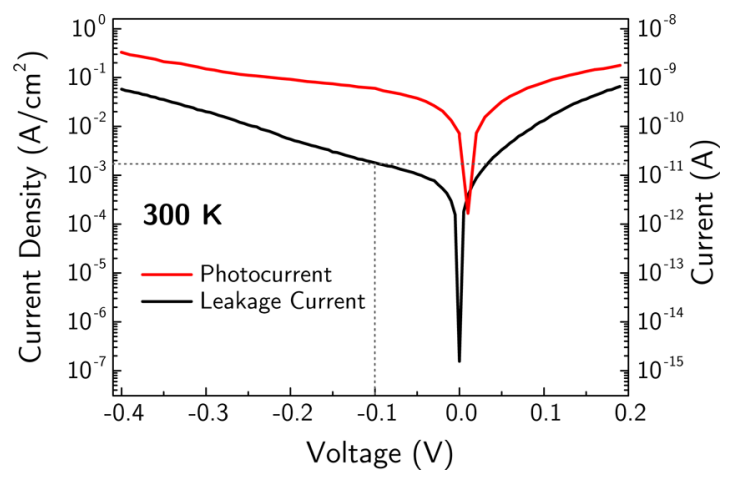

Figure 4. Semilog current-voltage characteristic of $\operatorname{InAs}_{0.93} \mathrm{Sb}_{0.07} p-i-$ $n$ photodiode at $300 \mathrm{~K}$ with a nanowire diameter of $80 \mathrm{~nm}$ and a length of $1.7 \mu \mathrm{m}$. The black and red plots show the leakage current and photocurrent density, respectively, for 200 contacted nanowires. At a typical operational bias of $-0.1 \mathrm{~V}$ the leakage current is $18 \mathrm{pA}$ which corresponds to a leakage current density around $2 \mathrm{~mA} / \mathrm{cm}^{2}$. For photocurrent measurements, illumination was provided by a $1.55 \mu \mathrm{m}$ laser to which the substrate is transparent.

current density below $2 \mathrm{~mA} / \mathrm{cm}^{2}$ for the 200 nanowires contacted by the metal. This result shows the nanowire photodetector to have significantly lower noise due to leakage current compared to conventional $\operatorname{InAs}(\mathrm{Sb})$ detectors, despite being grown on a heavily mismatched substrate.

The forward bias current can be fitted well as an ideal diode, with an ideality factor of 1.4 , and a simple series resistance. This indicates that the current-voltage characteristic is dominated by a single type-junction. Furthermore, the series resistance fitted is 2 orders of magnitude lower than the dynamic resistance in reverse bias, at $-0.1 \mathrm{~V}$, confirming that it cannot be the cause of the exceptionally low reverse leakage current density. Photocurrent was measured under illumination by a 14 $\mathrm{mW} 1.55 \mu \mathrm{m}$ laser; however, due to the continuous, optically opaque top contact covering the nanowire array, this was not directly incident on the nanowires. Instead it was fiber coupled onto an area adjacent to the edge of the contact, from where a small fraction was coupled into the wires through scattering and interface reflections. As seen in Figure 4, a photocurrent was detected which indicates that the nanowires are optically active; however, due to the optically opaque top and bottom contacts, the nanowires can only be indirectly illuminated at this time, and hence it is not yet possible to determine the quantum efficiency. Prior studies have been made of InAsSb nanowires with higher $\mathrm{Sb}$ fractions, configured for infrared photodetection. Without any type of junction these photoconductive wires showed some response; however, despite cooling to $5 \mathrm{~K}$, leakage remained in the $\mathrm{mA}$ range for $\sim 8000$ wires. $^{21}$

The $300 \mathrm{~K}$ peak spectral response in Figure 5 is significantly shorter than the photoluminescence (PL) peak which we attribute to the wavelength and wire diameter dependence of the HE11 mode in the nanowires. ${ }^{21}$ Increasing the absorption strength for longer wavelengths can be achieved by optimizing the nanowire diameter and since our aim is to detect in the mid-infrared, this will be the focus of future work. For PL measurements, undoped nanowires were grown with the dense pattern shown in Figure 1a containing an initial $200 \mathrm{~nm}$ InAs section followed by $800 \mathrm{~nm}$ of InAsSb. The overall PL peak, see Figure 5, consists of emission from both the InAs and InAsSb sections at 2850 and $3240 \mathrm{~nm}$, respectively. The emission from the InAs is blue-shifted from the expected wavelength by 


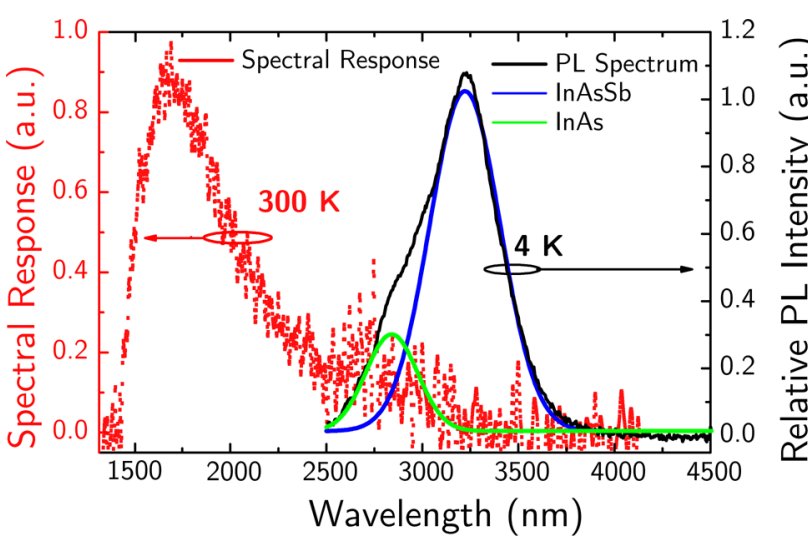

Figure 5. Spectral response at $300 \mathrm{~K}$ and $-0.1 \mathrm{~V}$ bias (red) and $4 \mathrm{~K}$ photoluminescence spectrum (black) of $\operatorname{InAs} \mathrm{s}_{0.93} \mathrm{Sb}_{0.07}$ nanowires. The PL spectrum is deconvoluted using two Gaussian peaks to show the contributions of the InAs (green) and InAsSb (blue) sections of the wire. The noise in the spectral response between 2500 and $3000 \mathrm{~nm}$ is an artifact of the system response correction due to atmospheric absorption.

around $5 \mathrm{meV}$ which is consistent with confinement in a $80 \mathrm{~nm}$ 1D quantum wire. ${ }^{22,23}$

Optimisation of the length and diameter of InP nanowires has been shown to increase the absorbance of nanowire arrays to levels which equal planar devices despite having only a fraction $(10 \%)$ of the volume. ${ }^{24}$ Assuming equal absorbance, $10 \%$ nanowire coverage, and the observed leakage current density reduction of 50 times, InAsSb nanowire photodetectors could increase the SNR by up to 500 times over conventional $\mathrm{InAs}(\mathrm{Sb})$ photodetectors.

InAs based photodiodes have long been known to suffer from high levels of surface leakage originating from an electron accumulation layer, such that, as the perimeter to area ratio increases, surface leakage tends to dominate the total leakage current. ${ }^{5}$ The perimeter/area ratio of the nanowires in this work is extremely high at $5 \times 10^{5} \mathrm{~cm}^{-1}$. Ker et al. separated surface and bulk leakage in InAs photodiodes and reported them as a function of temperature. ${ }^{5}$ Comparison with these results shows that conventional InAs photodiodes with the nanowire's dimensions would indeed be strongly dominated by surface leakage. Only by cooling to $125 \mathrm{~K}$ would this surface leakage current fall to the levels of the total leakage measured in these nanowires at $300 \mathrm{~K}$. Defining a mesa diode requires the removal of material through chemical etching, which disrupts the lattice and allows impurities to be introduced. By using monolithically grown nanowires to define the detecting volume, the surfaces remain unmodified and defect free which we believe leads to a reduction in the surface contribution to the overall leakage current. Even if surface leakage is neglected, cooling to $230 \mathrm{~K}$ would be required for the best InAs photodiodes to match the leakage current density measured in the nanowires at $300 \mathrm{~K}$.

Bulk leakage current density is closely associated with the crystalline quality of the detector material and hence it is inferred that the nanowire material exceeds the quality of conventional epitaxial $\operatorname{InAs}(\mathrm{Sb})$. This is supported by TEM analysis, which shows twinning of the zinc-blende lattice, but an absence of threading defects. The nanoscale diameter of the wires ensures that any $60^{\circ}$ threading dislocations originating from the interface with the (111) $\mathrm{Si}$ reach a sidewall and terminate within the lower $150 \mathrm{~nm}$ of the wire. Hence the crystalline structure of the wire in the region of the $p-i-n$ junction is expected, and observed, to be entirely free from any leakage-causing defects.

In summary, we have fabricated shortwave infrared photodetectors, using site-controlled nanowires on silicon, with unprecedentedly low leakage current at room temperature. Not only has this allowed for high quality shortwave infrared photodetectors to be grown on silicon substrates, but surprisingly, the leakage current density is almost 2 orders of magnitude lower than state of the art $\operatorname{InAs}(\mathrm{Sb})$ photodiodes. The unique design also allows for electrical conduction through the substrate which simplifies many aspects of the fabrication and expands the opportunities for integration. Although the nanowires occupy only a small fraction of light collection area, optimization of the length and diameter ${ }^{24,25}$ can significantly increase the absorbance up to that of planar films. ${ }^{2,24}$ Combined with the reduction in leakage current density over $\operatorname{InAs}(\mathrm{Sb})$, this should provide a more than 2 orders of magnitude increase in SNR over state of the art $\operatorname{InAs}(\mathrm{Sb})$ devices. This result is the first reported InAsSb nanowire photodiode and the lowest leakage current density reported for any $\operatorname{InAs}(\mathrm{Sb})$ photodiode at $300 \mathrm{~K}$. We believe that this represents a major step toward high-performance mid-infrared photodetectors compatible with silicon technologies and which can be integrated with other photonic systems.

Methods. Nanowire Growth. The nanowires were grown on boron-doped (111) orientated Si substrates with a known oxide thickness of either 20 or $50 \mathrm{~nm}$. The substrates were spincoated with PMMA and then patterned using electron beam lithography using a hole diameter of $50 \mathrm{~nm}$. After development the substrates were wet etched using 10:1 buffered oxide etch (BOE) to remove the oxide within the exposed holes. The resist was then removed, and the substrates were dipped in BOE once more to ensure a stable surface ${ }^{26}$ before being loaded immediately into the MBE reactor. The nanowires were grown using a VG V80h MBE reactor and 2D growth rates for In, As, and $\mathrm{Sb}$ were calibrated using (001) InAs, GaAs, and $\mathrm{InSb}$, respectively. The In $2 \mathrm{D}$ growth rate was set to $0.07 \mathrm{ML} / \mathrm{s}$ with As at $1.8 \mathrm{ML} / \mathrm{s}$ and $\mathrm{Sb}$ at $1.15 \mathrm{ML} / \mathrm{s}$ which gives a V-III ratio of $42: 1$. The substrate was initially heated to $600{ }^{\circ} \mathrm{C}$ without any group $\mathrm{V}$ flux before being cooled to the growth temperature of $480{ }^{\circ} \mathrm{C}$. The nanowire growth was then initiated by simply opening the In, As, and Sb shutters. All samples in this report are grown with the same $\mathrm{Sb}$ composition. Dopant cell temperatures were set according to the measured concentrations in $2 \mathrm{D} \mathrm{InSb}$ of $7 \times 10^{17} \mathrm{~cm}^{-3}$ for Be and $5 \times$ $10^{17} \mathrm{~cm}^{-3}$ for Te.

Photodetector Fabrication. To fabricate the photodetectors, the substrates were spin coated with $2 \mu \mathrm{m}$ thick SU-8 and flood exposed to harden the resist. The resist was then etched using reactive ion etching in $\mathrm{CF}_{4}$ and $\mathrm{O}_{2}$ to expose the tips of the wires. The wire tips were then contacted using $30 \mathrm{~nm} \mathrm{Ti}$ and $200 \mathrm{~nm} \mathrm{Au}$ with $200 \mathrm{~nm} \mathrm{Al}$ on the back of the substrate forming the back contact. The schematic of a finished device is shown in Figure 3a showing the different sections of the nanowire.

Spectral Response and Current Voltage Measurements. Spectral response measurements on the photodetectors were performed using a Bruker Vertex FTIR at room temperature and a bias of $-0.1 \mathrm{~V}$.

Leakage current measurements were carried out using a Lakeshore TTPX probe station with guarded triaxial connectors and a Keithley 6430 Sub-Femtoamp SourceMeter. A source 
delay of $10 \mathrm{~s}$ was used to prevent transient triboelectric or piezoelectric effects from influencing the measurement. The measured leakage current at room temperature, shown in Figure 4, at a typical operational bias of $-0.1 \mathrm{~V}$ was $18 \mathrm{pA}$. However, due to variation in nanowire length, not all of the wires were exposed after etching back the resist.

To determine the number of wires contacted, we used SEM to assess the fraction of wires exposed after etching. The metal contact covered a portion of a large low density nanowire array with a pitch of $2 \mu \mathrm{m}$. Areas of nanowires adjacent to the contact were imaged with the image field of view covering approximately 230 nanowires. Two images of this type were taken, and the number of nanowires which protruded above the surface of the SU-8 were counted in both images to determine the fraction which were exposed. (See Supplementary Figure 2 for an example of both an exposed and a covered nanowire.) Using this method we determined that approximately $15 \%$ of the wires were exposed. The metal contact covered an area containing around 1500 wires, which would mean that approximately 200 wires were contacted by the metallization. The cross-sectional area was estimated using the nanowire diameter and approximating to a circular cross-section.

Photoluminescence. The sample was cooled in a continuous flow liquid helium cryostat to $4 \mathrm{~K}$ and excited with a $808 \mathrm{~nm}$ laser with excitation power of $150 \mathrm{~mW}$. The spectrum was recorded using a Bentham TMc300 monochromator with InSb detector.

Transmission Electron Microscopy. Annular dark field (ADF) scanning transmission electron microscopy (STEM) images were taken in an aberration-corrected JEOL ARM-200F microscope working at $200 \mathrm{kV}$. Focused ion beam (FIB) liftout TEM specimens to investigate the interface with the substrate were prepared using a JEOL JIB4500.

\section{ASSOCIATED CONTENT}

\section{S Supporting Information}

The Supporting Information is available free of charge on the ACS Publications website at DOI: 10.1021/acs.nanolett.5b03449.

Figure of energy-dispersive X-ray (EDX) spectrum of InAsSb nanowire on Si substrate (PDF)

Figure of exposed nanowire after etching back SU-8 (PDF)

\section{AUTHOR INFORMATION}

\section{Corresponding Authors}

*E-mail: m.thompson@lancaster.ac.uk.

*E-mail: a.r.marshall@lancaster.ac.uk.

\section{Author Contributions}

M.D.T., A. Alhodaib, and A.R.J.M. conceived the project, designed the arrays, and performed the MBE growth. A. Aziz patterned and developed the wafers before growth. M.D.T. fabricated the photodetectors. A.R performed the AFM analysis. A.P.C. and M.D.T. performed the spectral response and current-voltage measurements. A. Alhodaib and A.K. were responsible for photoluminescence. J.S. and L.W. provided Xray analysis. A.M.S. provided TEM analysis. M.D.T. and A.R.J.M. cowrote the paper.

\section{Funding}

M.D.T and A.R.J.M. supported by DSTL under the project CDE 65947, and A.R.J.M. additionally supported by Royal Academy of Engineering grant number EP/H043 993/1. A.K. supported by EU Marie Curie ITN PROMIS grant agreement No. H2020-MSCA-ITN-2014-641899. J.S. and L-E.W. have been supported by the Swedish Research Council, the Swedish Foundation for Strategic Research, and the European Union seventh Framework Program E2SWITCH (Grant Agreement No. 619509). A.R. supported by EPSRC.

\section{Notes}

The authors declare no competing financial interest.

\section{ACKNOWLEDGMENTS}

The authors thank DSTL for funding under the project CDE 65947 and the Royal Academy of Engineering for the fellowship awarded to A. Marshall (grant number EP/H043 993/1). A.M. Sanchez thanks S.Hindmarsh for the technical support in the focus ion beam specimen preparation. All the data used for the plots in this work are publicly available at: http://dx.doi.org/10.17635/lancaster/researchdata/51.

\section{REFERENCES}

(1) Tomioka, K.; Tanaka, T.; Hara, S.; Hiruma, K.; Fukui, T. III-V Nanowires on Si Substrate: Selective-Area Growth and Device Applications. IEEE J. Sel. Top. Quantum Electron. 2011, 17, 11121129.

(2) Sourribes, M. J. L.; Isakov, I.; Panfilova, M.; Liu, H.; Warburton, P. A. Mobility Enhancement by Sb-mediated Minimisation of Stacking Fault Density in InAs Nanowires Grown on Silicon. Nano Lett. 2014, 14, 1643-1650. PMID: 24502770

(3) Ertekin, E.; Greaney, P. A.; Chrzan, D. C.; Sands, T. D. Equilibrium limits of coherency in strained nanowire heterostructures. J. Appl. Phys. 2005, 97, 114325.

(4) Krishnamachari, U.; Borgstrom, M.; Ohlsson, B. J.; Panev, N.; Samuelson, L.; Seifert, W.; Larsson, M. W.; Wallenberg, L. R. Defectfree $\mathrm{InP}$ nanowires grown in [001] direction on InP (001). Appl. Phys. Lett. 2004, 85, 2077-2079.

(5) Ker, P.; Marshall, A.; Krysa, A. B.; David, J.; Tan, C. H. Temperature Dependence of Leakage Current in InAs Avalanche Photodiodes. IEEE J. Quantum Electron. 2011, 47, 1123-1128.

(6) Maddox, S. J.; Sun, W.; Lu, Z.; Nair, H. P.; Campbell, J. C.; Bank, $S$. R. Enhanced low-noise gain from InAs avalanche photodiodes with reduced dark current and background doping. Appl. Phys. Lett. 2012, 101,151124

(7) Shao, H.; Li, W.; Torfi, A.; Moscicka, D.; Wang, W. Roomtemperature InAsSb photovoltaic detectors for mid-infrared applications. IEEE Photonics Technol. Lett. 2006, 18, 1756-1758.

(8) Weiss, E.; Klin, O.; Grossmann, S.; Snapi, N.; Lukomsky, I.; Aronov, D.; Yassen, M.; Berkowicz, E.; Glozman, A.; Klipstein, P.; Fraenkel, A.; Shtrichman, I. InAsSb-based XBnn bariodes grown by molecular beam epitaxy on GaAs. J. Cryst. Growth 2012, 339, 31-35.

(9) Wagner, R. S.; Ellis, W. C. Vapor-liquid-solid Mechanism Of Single Crystal Growth. Appl. Phys. Lett. 1964, 4, 89-90.

(10) Mandl, B.; Stangl, J.; Martensson, T.; Mikkelsen, A.; Eriksson, J.; Karlsson, L. S.; Bauer, G.; Samuelson, L.; Seifert, W. Au-Free Epitaxial Growth of InAs Nanowires. Nano Lett. 2006, 6, 1817-1821. PMID: 16895379

(11) Brotherton, S. D.; Lowther, J. E. Electron and Hole Capture at $\mathrm{Au}$ and Pt Centers in Silicon. Phys. Rev. Lett. 1980, 44, 606-609.

(12) Anyebe, E.; Rajpalke, M.; Veal, T.; Jin, C.; Wang, Z.; Zhuang, Q. Surfactant effect of antimony addition to the morphology of selfcatalyzed InAsSb nanowires. Nano Res. 2015, 8, 1309-1319.

(13) Austin, M. D.; Ge, H.; Wu, W.; Li, M.; Yu, Z.; Wasserman, D.; Lyon, S. A.; Chou, S. Y. Fabrication of $5 \mathrm{~nm}$ linewidth and $14 \mathrm{~nm}$ pitch features by nanoimprint lithography. Appl. Phys. Lett. 2004, 84, 52995301.

(14) Munshi, A. M.; Dheeraj, D. L.; Fauske, V. T.; Kim, D. C.; Huh, J.; Reinertsen, J. F.; Ahtapodov, L.; Lee, K. D.; Heidari, B.; van Helvoort, A. T. J.; Fimland, B. O.; Weman, H. Position-Controlled 
Uniform GaAs Nanowires on Silicon using Nanoimprint Lithography. Nano Lett. 2014, 14, 960-966. PMID: 24467394

(15) Joyce, H.; Gao, Q.; Wong-Leung, J.; Kim, Y.; Tan, H.; Jagadish, C. Tailoring GaAs, InAs, and InGaAs Nanowires for Optoelectronic Device Applications. IEEE J. Sel. Top. Quantum Electron. 2011, 17, $766-778$.

(16) Hertenberger, S.; Rudolph, D.; Bichler, M.; Finley, J. J.; Abstreiter, G.; Koblmuller, G. Growth kinetics in position-controlled and catalyst-free InAs nanowire arrays on $\mathrm{Si}(111)$ grown by selective area molecular beam epitaxy. J. Appl. Phys. 2010, 108, 114316.

(17) Johansson, J.; Bolinsson, J.; Ek, M.; Caroff, P.; Dick, K. A. Combinatorial Approaches to Understanding Polytypism in IIIV Nanowires. ACS Nano 2012, 6, 6142-6149. PMID: 22681568

(18) Thelander, C.; Caroff, P.; Plissard, S.; Dey, A. W.; Dick, K. A. Effects of Crystal Phase Mixing on the Electrical Properties of InAs Nanowires. Nano Lett. 2011, 11, 2424-2429. PMID: 21528899

(19) Xu, T.; Dick, K. A.; Plissard, S.; Nguyen, T. H.; Makoudi, Y.; Berthe, M.; Nys, J.-P.; Wallart, X.; Grandidier, B.; Caroff, P. Faceting, composition and crystal phase evolution in IIIV antimonide nanowire heterostructures revealed by combining microscopy techniques. Nanotechnology 2012, 23, 095702.

(20) Murayama, M.; Nakayama, T. Chemical trend of band offsets at wurtzite/zinc-blende heterocrystalline semiconductor interfaces. Phys. Rev. B: Condens. Matter Mater. Phys. 1994, 49, 4710-4724.

(21) Svensson, J.; Anttu, N.; Vainorius, N.; Borg, B. M.; Wernersson, L.-E. Diameter-Dependent Photocurrent in InAsSb Nanowire Infrared Photodetectors. Nano Lett. 2013, 13, 1380-1385. PMID: 23464650

(22) Koblmuller, G.; Hertenberger, S.; Vizbaras, K.; Bichler, M.; Bao, F.; Zhang, J.-P.; Abstreiter, G. Self-induced growth of vertical freestanding InAs nanowires on $\mathrm{Si}(111)$ by molecular beam epitaxy. Nanotechnology 2010, 21, 365602.

(23) Koblmuller, G.; Vizbaras, K.; Hertenberger, S.; Bolte, S.; Rudolph, D.; Becker, J.; Doblinger, M.; Amann, M.-C.; Finley, J. J.; Abstreiter, G. Diameter dependent optical emission properties of InAs nanowires grown on Si. Appl. Phys. Lett. 2012, 101, 053103.

(24) Anttu, N.; Abrand, A.; Asoli, D.; Heurlin, M.; Aberg, I.; Samuelson, L.; Borgstrom, M. Absorption of light in InP nanowire arrays. Nano Res. 2014, 7, 816-823.

(25) Foldyna, M.; Yu, L.; Roca i Cabarrocas, P. Theoretical shortcircuit current density for different geometries and organizations of silicon nanowires in solar cells. Sol. Energy Mater. Sol. Cells 2013, 117, 645-651. Dye Sensitized Solar Cells, Organic, Hybrid Solar Cells and New Concepts Dye Sensitized Solar Cells, Organic, Hybrid Solar Cells and New Concepts.

(26) Higashi, G. S.; Chabal, Y. J.; Trucks, G. W.; Raghavachari, K. Ideal hydrogen termination of the Si (111) surface. Appl. Phys. Lett. 1990, 56, 656-658. 